\title{
THE VISUAL MECHANISMS OF \\ TENEBRIO MOLITOR: CHANGES IN THE ELECTRO- RETINOGRAM AS FUNCTION OF THE STIMULUS DURATION
}

\author{
BY U. YINON* \\ The Vison Research Laboratory, Hadassah University Hospital, \\ Ferusalem, Israel
}

(Received 27 October 1970)

INTRODUCTION

The yellow mealworm beetle Tenebrio molitor represents a group of insects living almost constantly under conditions of darkness. Some evolutionary changes presumably occurred in the visual mechanism of this species during or after the adoption of its specific habitat. Various aspects of its visual mechanism have already been investigated (Yinon, 1969, 1970a; Yinon and Auerbach, 1969). In the present studies another aspect of the visual mechanism - the response as function of stimulus duration - has been investigated. This has been compared with the response in diurnal or nocturnal insects not living in a permanently dark habitat.

The insect electroretinogram (ERG) is a complex of potential changes. Hartline (1928) partially explained the origin of the various components by demonstrating that the negative component of the insect ERG comes from the retina, while the positive component arises from the optic ganglion. The relative prominence of these two components varies with the anatomy of the eye. Therefore, in comparison to the vertebrate's ERG (Davson, 1962), there is less certainty about the insect's ERG pattern (Wigglesworth, 1965; Yinon, 1970b). In the present studies the origin of various components of the ERG have been studied as well as their relative contribution in the context of the duration and intensity of the stimulus.

\section{METHODS}

Results were calculated for 23 adults of the yellow mealworm beetle $T$. molitor (L.). The animals were dark-adapted for 5-10 min before each experiment. Between stimuli of different durations at each intensity, intervals of one minute were allowed for recovery in the dark, and between series of different intensities the intervals were of some minutes. Only one intensity level $(\log I=0 \cdot 0)$ was used when exposures of longer than I $\mathrm{s}$ were given and the intervals were of $2-3 \mathrm{~min}$ between the stimuli.

The optical system was described previously (Yinon, 1970c). Exposures from I ms to Is were given by Wollensak shutter and from I ms to $14 \mathrm{~s}$ by Exacta shutter. The signals from these shutters were displayed on the oscilloscope for each ERG. Relative

- Present address: Division of Biology, California Institute of Technology, Pasadena, California 9 Irog. 

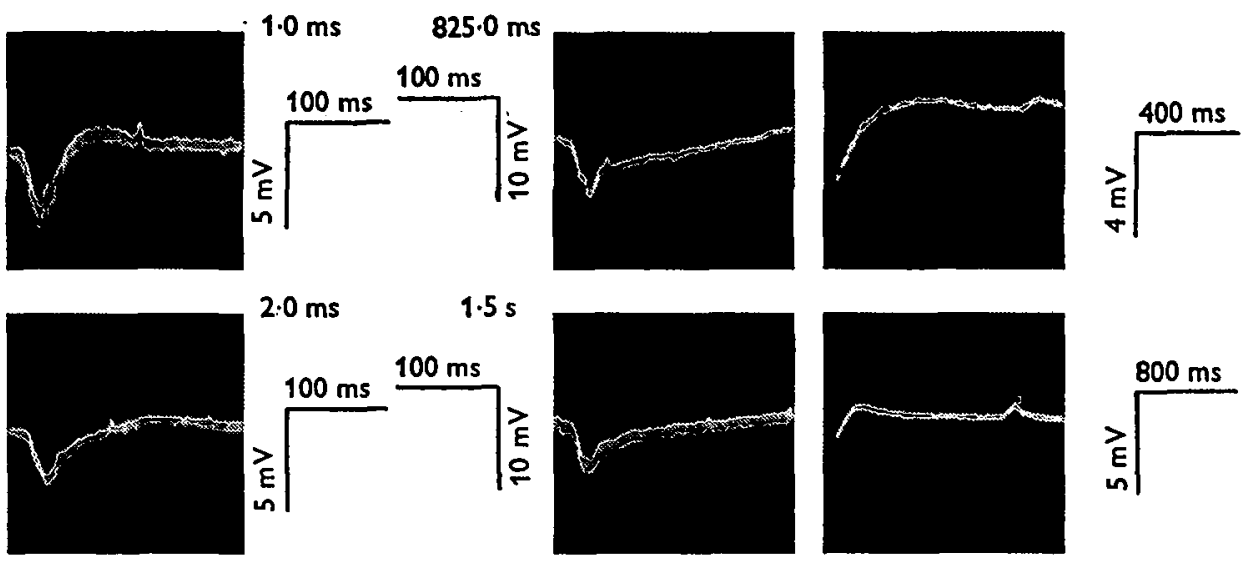

\section{$2: 0 \mathrm{~ms}$}

$1.5 \mathrm{~s}$
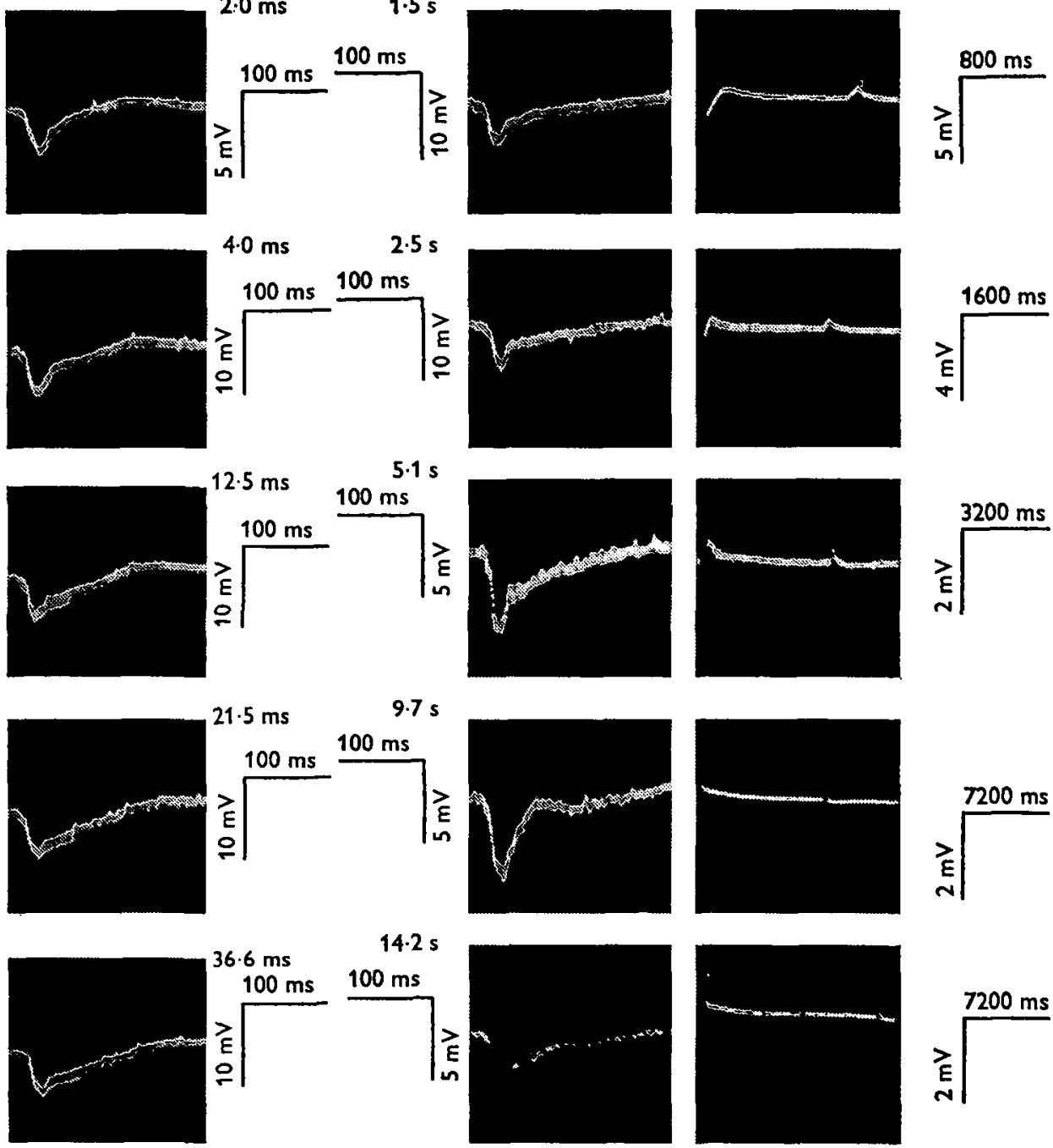

1

II

Fig. 1 . Changes in the ERG for a wide range of stimulus durations at a given intensity ( $\log I=0.0$ ). Stimulus durations for Group I are seen above the first column of calibration marks and for Group II above the second one. The left column of responses in Group II shows the 'on' potential of the same responses as the right column but on a different time scale.

The ERG for a stimulus duration of $\mathrm{r} \cdot 0 \mathrm{msec}$ has a biphasic pattern including 'on' negative and a gradual positive wave. The ERG for $14^{\prime 2} \mathrm{sec}$ includes a faster positive component after the 'on', positive 'off' and a small negative wave after the 'off'. 
light intensity was determined by Ilford neutral circular wedge $(3.0 \mathrm{log}$ units) and y Kodak optical density filters. Schott heat filter was used to exclude infrared radianon. The maximum intensity $(\log I=0.0)$ was 70 lux over the eye.

The animal was connected through a pre-amplifier to the recording system which was synchronized with the shutter oscilloscope and the camera. The spontaneous activity was monitored by an additional oscilloscope. In some of the experiments an averaging device (computer of Average Transients, $400 \mathrm{~B}$, TMC), connected to the electronic counter was used and was triggered by a photo-cell placed near the eye.

For other experimental conditions see Yinon and Auerbach (1969) and Yinon $(1970 b)$.

\section{RESULTS}

The ERG pattern for short stimulus durations (less than $100 \mathrm{msec}$ ) is composed of a fast negative potential which is followed by a slow positive one. In longer durations a faster positive component appeared coupled with the end of the stimulus, thus called

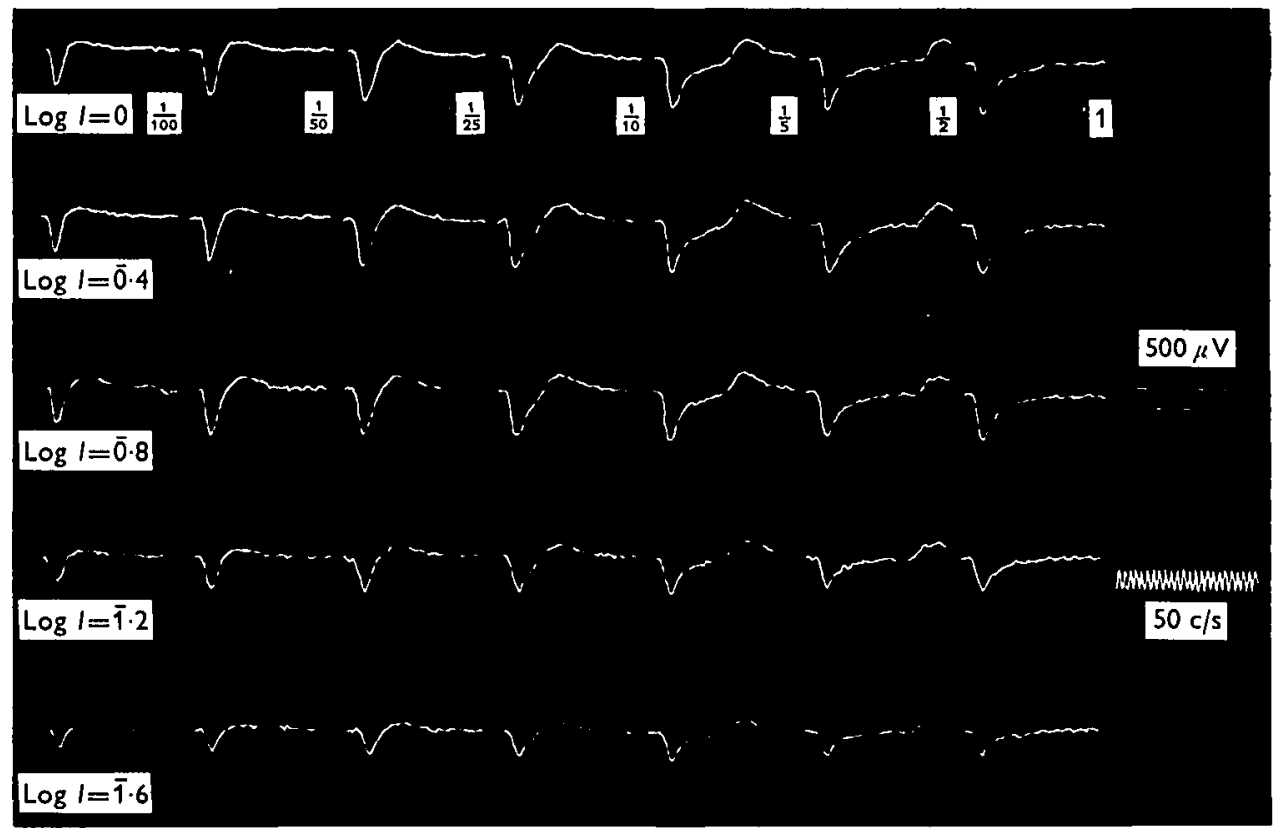

Fig. 2. The combined effect of stimulus duration and intensity on the ERG.

the 'off-effect' (Figs. I, 2). When stimuli were sequentially increased the 'off' is seen to separate out gradually from the ERG complex. The separation begins in the same exposure time (100 msec) at different intensities, i.e. it depends on the stimulus duration only. The 'off' potential is followed by a small gradual negative wave. A negative component was not found before the 'off' wave as in many diurnal and nocturnal species (Goldsmith, 1963; Mazokhin-Porshnyakov, 1969; Yinon, 1970d). After the separation phenomenon takes place the first negative potential is seen clearly to be the 'on-effect'. When the eye was exposed to stimuli longer than I sec the positive dow potential appeared after the 'on' increased (Fig. I). 
For a given intensity and above a certain threshold of stimulus duration, the exposure time and the latent period of the 'on' are independent. It is not cleay however, why the latency and the peak latency (the period from the beginning of the stimulus to the appearance of the response peak) are still constant in their length in

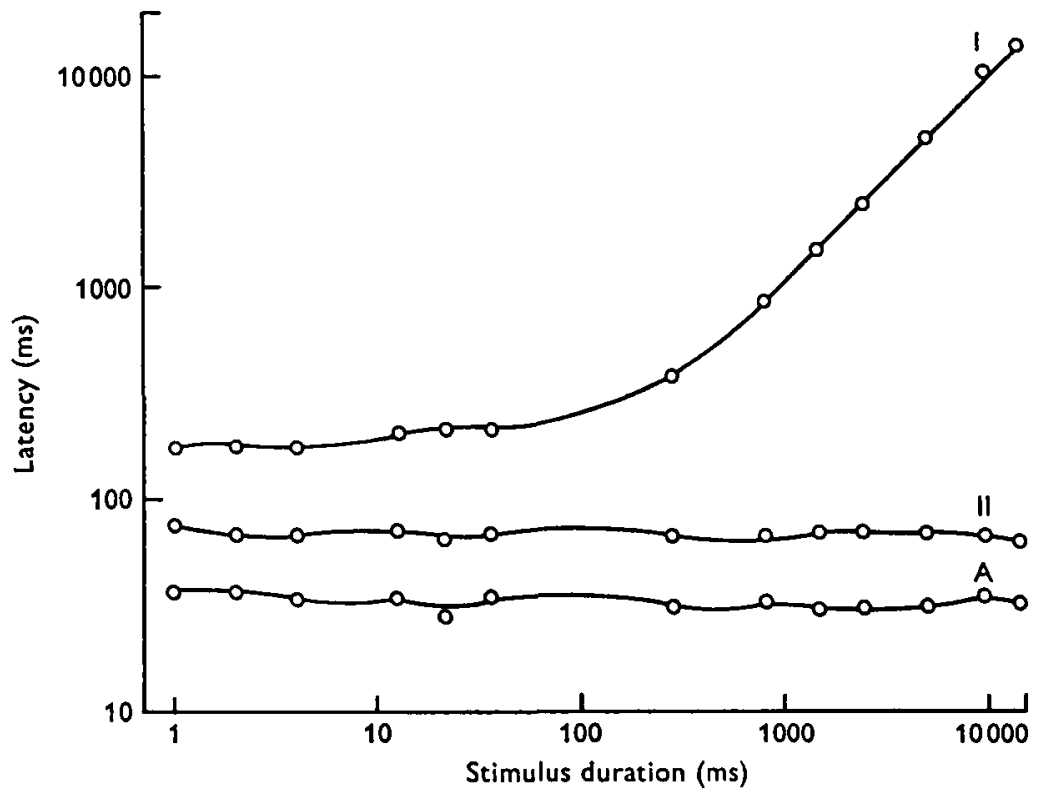

Fig. 3. The latent periods of the ERG components as functions of stimulus duration ( $\mathrm{I}=$ 'off', II = 'on'-peak, $A=$ 'on').

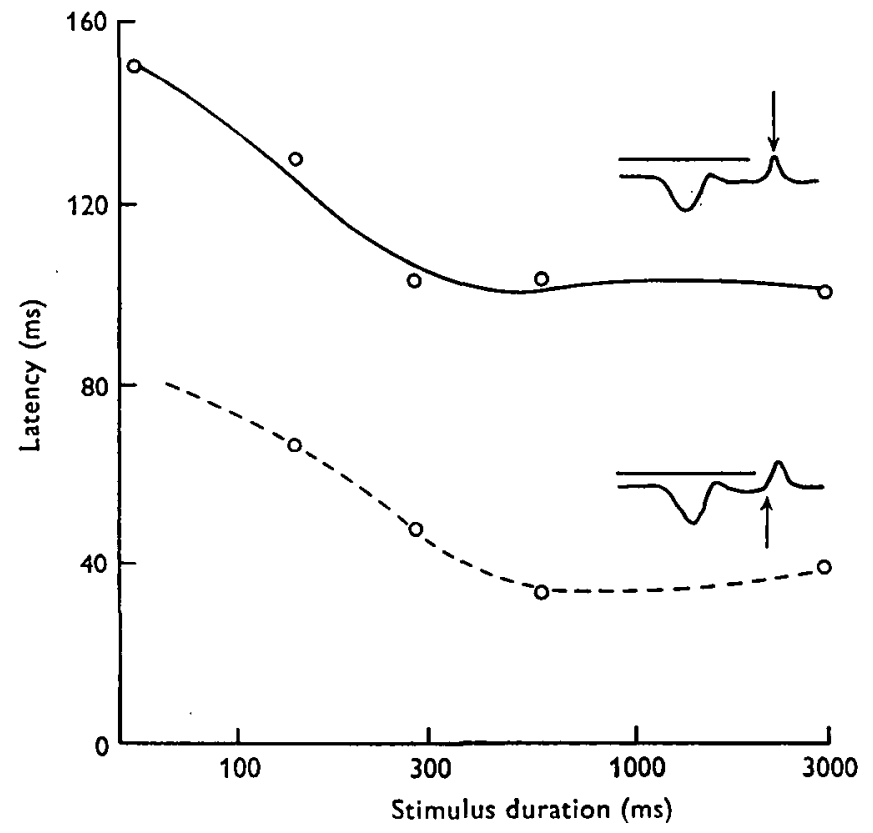

Fig. 4. 'Off' latencies (as measured after the extinction of the stimulus) as function of the stimulus duration. 
Table I. Changes in the latency of the 'off'-effect after the beginning and after the ending of the light stimulation

$\begin{array}{ccc}\begin{array}{c}\text { Stimulus } \\ \text { duration } \\ (\mathrm{ms})\end{array} & \overbrace{\begin{array}{c}\text { From } \\ \text { beginning }\end{array}}^{\text {'Off' latency }} \begin{array}{c}\text { From } \\ \text { ending }\end{array} \\ 55.0 & & \\ 140.2 & 206.4 & 66.2 \\ 283.3 & 330.6 & 47.3 \\ 584.2 & 617.1 & 32.9 \\ 2966.6 & 3006.1 & 39.5\end{array}$

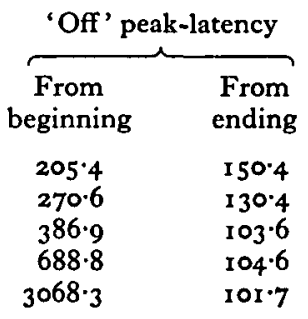
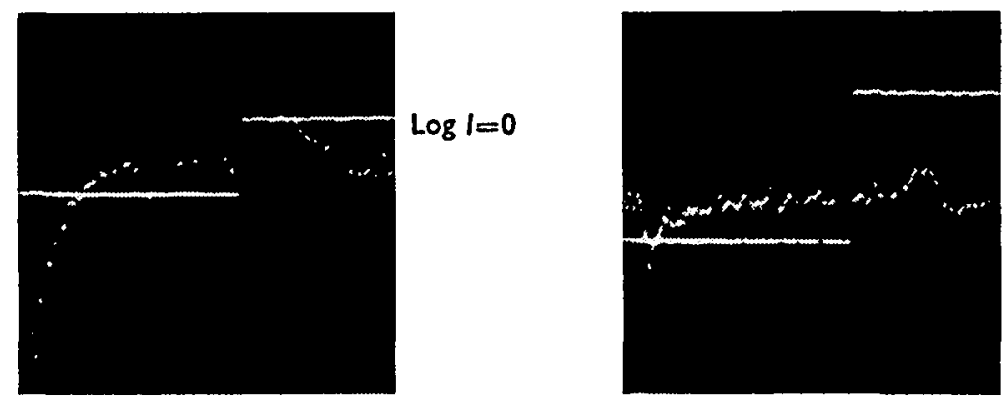

$\log I=-1 \cdot 2$
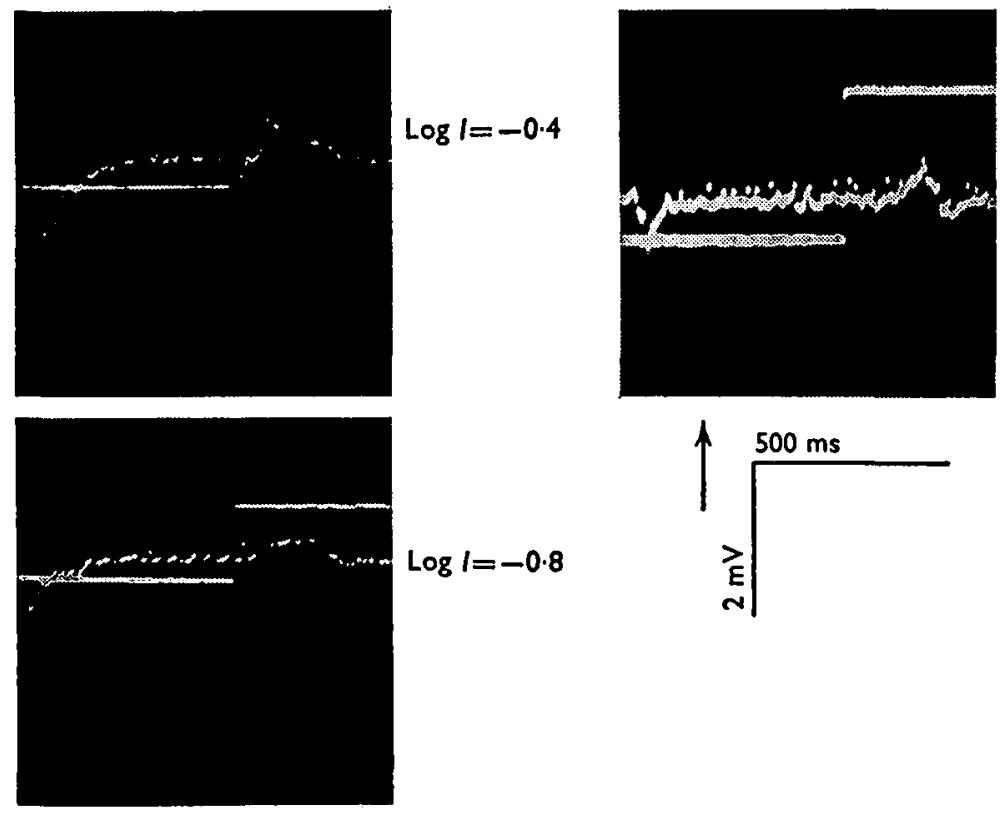

$\log I=-1.6$

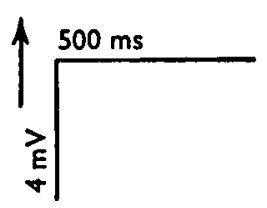

Fig. 5. The effect of various intensities on the ERG at a given stimulus duration. Averaged responses are seen. The lower trace in each picture shows the beginning and ending of the stimulus. 
presence of subthreshold stimuli (Fig. 3). The latent period of the 'off' is clearly related to the stimulus extinction, yielding a linear ratio (as function of duration) an stimulus durations longer than 300 msec (Fig. 3). But in short stimulus durations thflexibility in the length of the 'off' latency is higher since its appearance is not tightly coupled with the end of the stimulus. The 'off' latency decreased - after stimulus

Table 2. Changes in the $E R G$ as function of the light intensity at a given exposure time $(590 \mathrm{~ms})$. (Latencies were measured from the beginning of the light stimulus)

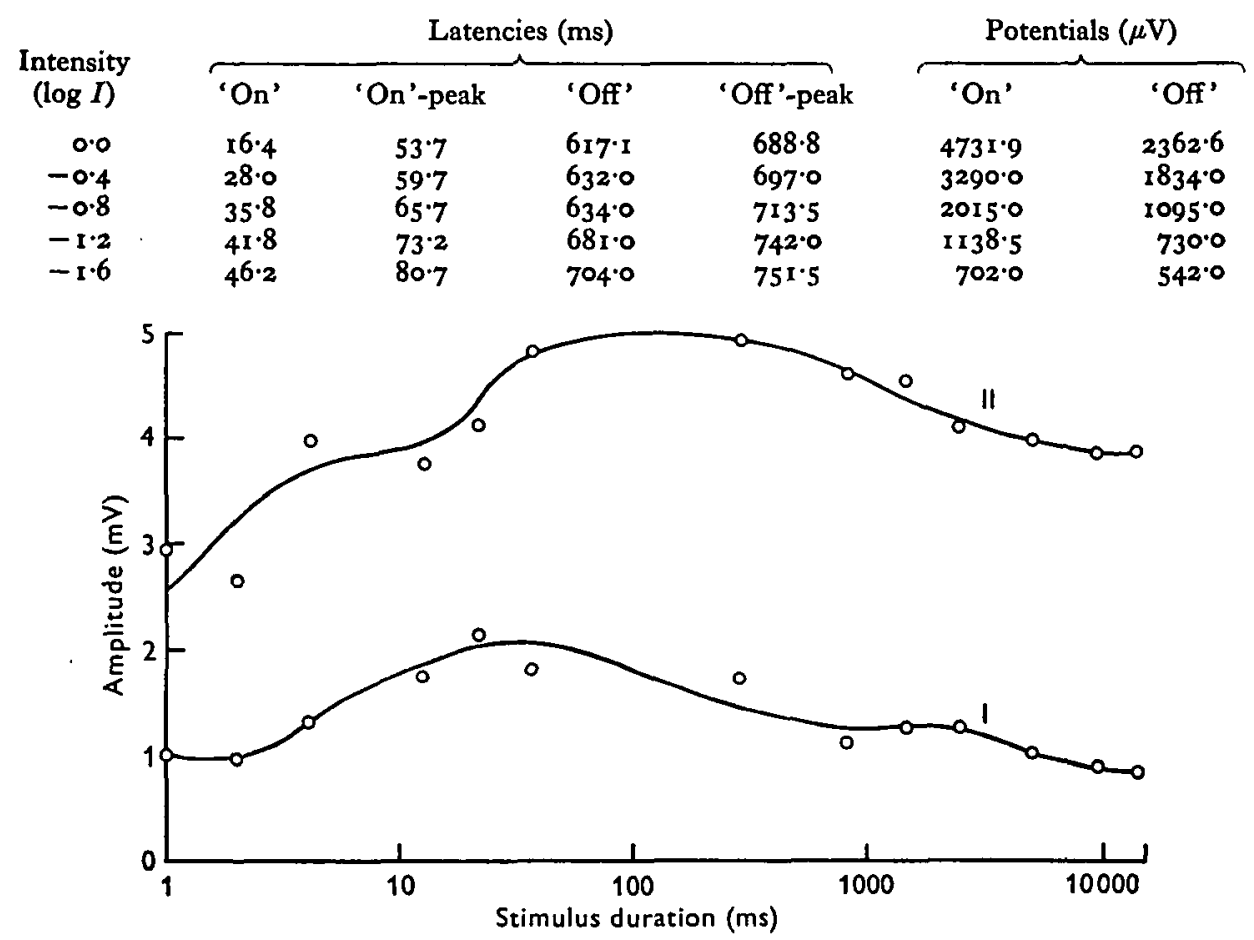

Fig. 6. The ERG amplitude as function of the stimulus duration ( $I=$ 'off', II $=$ 'on').

extinction - when the stimulus duration increased up to values of $500 \mathrm{msec}$ (Fig. 4, Table I). In exposures longer than this the 'off' latencies are not more influenced by the addition of light energy to the stimulus. Thus it reacts differently than the 'on' under the same conditions. The difference between the latent period $(70 \mathrm{msec}$, $\log I=0.0$ ) and the peak latency $(150 \mathrm{msec}$ ) of the 'off' (after extinction of the stimulus) is constant.

The combined influence of stimulus duration and stimulus intensity was also studied. The latency of 'on' and 'off' increased with the decreasing of the light intensity in a given exposure time (Figs. 2, 5; Table 2). If small intensity is provided $(\log I=-\mathrm{r} \cdot 6)$ in different durations, the 'on' latency was found to be very unstable in its length indicating a poor effectiveness of the visual system in this condition. When the 'off' latencies were plotted as function of the stimulus intensity and duration, it was found that the results are dependent on the intensity level and not on the combined effect of both parameters. 
The stimulus duration was found to determine the ERG magnitude to a given intensity as expected from Bunsen-Roscoe law. A similar trend is seen for both 'on' Tnd 'off' effects, although in the latter the response level is lower (Fig. 6). This and the similarity shown by the intensity functions presumably indicate a common origin in the receptor cells. When the set of the curves received for different stimulus durations under five intensity levels (from $\log I=0 \cdot 0$ to $\log I=-\mathrm{I} \cdot 6$ ) were compared (for 'on' effect) equal amplitudes were received in combination of stimuli ranged from ro-40 msec duration and intensities between $\log I=-0.4$ to $\log I=-\mathbf{I} \cdot 2$. At higher intensities the response amplitude was found to depend on the light intensity only. (Insufficient data were obtained to make it possible to calculate $I \times t[I=$ intensity, $t=$ duration]) constants.

\section{DISCUSSION}

Autrum (1958) has classified compound eyes of beetles among the 'slow type' giving negative monophasic electrical response. In contrast, $T$. molitor which belongs taxonomically to this group has a diphasic ERG including also an 'off' component. The ERG of the dragonfly Aeshna cyanea consists of a retinal and a neural component. The latter ('off' effect) is contributed by the optic ganglia (Autrum, 1958). The positive 'off' response of Tenebrio molitor seems to originate in the receptor zone as well as the 'on' response. By sequential and gradual increase of the stimulus duration it was possible to separate the ERG of T. molitor into 'on' and 'off' potentials (in $100 \mathrm{~ms}$ ). Among previous studies reviewed it was found to be in $330 \mathrm{~ms}$ for the stemmata of Bombyx mori (Ishikawa \& Hirao, 1960 ).

An increase of amplitude as function of stimulus duration has been observed in some species. It was found in the ERG of the cricket Tachycines asynamorus by Autrum (1948a) and for the 'off' potential (the 'on' remained constant) of the fly Calliphora erythrocephala (Autrum, 1948b). A peak occurred for the euphausiid Euphausia pacifica at a stimulus duration of roo ms (Boden \& Kampa, 1965) (as in Tenebrio molitor) and at $430 \mathrm{~ms}$ for the crayfish Procambarus clarkii (Naka \& Kuwabara, 1959). A constant response may be obtained with different intensities of illumination provided that the duration of the stimulus is varied in such a way that the product of intensity and duration is constant $(I \times t=$ const., Bunsen-Roscoe Law of photochemistry). In insects this relation was found to hold for exposures of less than $80 \mathrm{~ms}$ (Harline, 1928) whereas in man it is between 2 and $100 \mathrm{~ms}$ (LeGrand, 1957). It was found to be between 10 and $40 \mathrm{~ms}$ in the present investigation. Exposures longer than this yield a maximum of the principal potential, which depends on the intensity alone. According to the present studies the amplitude is diminished at a given intensity level in durations above $300 \mathrm{~ms}$ for a reason which is still unknown.

In a previous study a way was found to calculate the spectral response of $T$. molitor according to the latency ('on') function which is approximately a reciprocal of the amplitude (Yinon, 1969). In the present studies a relation was not found between the amplitude and the latency in relation to the stimulus duration. Thus the 'on' latency is no longer a function of the stimulus duration, even in the small range in which the amplitude is. 


\section{SUMMARY}

I. The ERG complex of the compound eye of the yellow mealworm bettle Tenebr molitor (L.) separates into two components with stimulus durations of longer than I $00 \mathrm{~ms}$. These are the 'on' and the 'off' effects. Above stimulus durations of is the positive potential following the 'on' is faster and the positive 'off' is followed by a small negative one.

2. The latent period of the 'on' is independent of the stimulus duration while for stimulus durations of longer than $300 \mathrm{~ms}$ the 'off' latency is coupled with the end of the stimulus. If measured after the extinction of the stimulus the 'off' latency is affected by the stimulus duration.

3. The 'on' and 'off' amplitudes behave similarly for various stimulus durations. Both originate in the receptor cells.

4. The Bunsen-Roscoe Law of photochemistry holds for stimulus durations of ro-40 ms. For a given intensity, the amplitudes diminished for durations longer than $300 \mathrm{~ms}$.

I am indebted to Professor E. Auerbach, Vision Research Laboratory, Hadassah University Hospital, Jerusalem, Israel, in whose laboratory this work was conducted, and also to Professor A. Shulov, Department of Entomology, the Hebrew University, who provided financial support.

\section{REFERENCES}

Autrum, H. (1948a). Zur Analyse des zeitlichen Auflösungsvermögens des Insektenauges. Nachr. Ges. Wiss. Göttingen, Math.-physik kl. 13-18.

Autrum, H. (1948b). Uber das zeitliche Auflösungsvermögen des Insektenauges. Nachr. Ges. Wiss. Göttingen, Math.-physik. kl. Biol.-Phys. Abt. 8-13.

AutRum, H. (1958). Electrophysiological analysis of the visual systems in insects. Exp. Cell. Res. (Suppl.) 5, 426-39.

Boden, B. P. \& KAMPA, E. M. (1965). An aspect of euphasiid ecology revealed by echo-sounding in a fjord. Crustaceana $9,155-73$.

Davson, H. (1962). The Eye, vol. 2. New York and London: Academic Press.

Goldsmith, T. H. (1963). The course of light and dark adaptation in the compound eye of the honeybee. Comp. Biochem. Physiol. 10, 227-37.

Hartline, H. K. (1928). A quantitative and descriptive study of the electric response to illumination of the arthropod eye. Am. F. Physiol. 83, 466-83.

Ishikawa, S. \& Hirao, T. (I960). Electrophysiological studies on vision of the silkworm Bombyx mori I: Electroretinogram of stemmata. F. Seric. Sci. Fapan 29, 8-14.

LEGrand, Y. (1957). Light, Colour and Vision. London: Chapman and Hall Ltd.

Mazokhin-Porshnyakov, G. A. (1969). Insect Vision. New York: Plenum Press.

Naka, K. \& Kuwabara, M. (I959). Two components of the compound eye of the crayfish. $\mathcal{F}$. Exp. Biol. 36, $5 \mathrm{I}-6 \mathrm{I}$.

YrNon, U. (1969). Spectral efficiency as function of latency in the visual mechanism of insect (Tenebrio molitor L.). Experientia 25, 711 .

YinON, U. \& AUERBACH, E. ( I969). The visual mechanisms of Tenebrio molitor: Variations taking place in the ERG of pupa and aduit during development. F. Exp. Biol. 5r, 635-41.

Yinon, U. (1970a). The visual mechanisms of Tenebrio molitor: Some aspects of the spectral response. F. Exp. Biol. 53, $221-9$.

YINON, U. (1970b). Similarity of the electroretinogram in insects. F. Insect Physiol. 16, 221-5.

YINON, U. (I970 c). The electrophysiological and behavioral response of a stored product beetle to light. F. Stored Prod. Res. 6, 195-8.

YINON, U. (1970d). Electrophysiological evidence of relationships in Acridid insects. J. System. Zool. x9, 397-8.

Wigglesworth, V. B. (1965). The Principles of Insect Physiology. London: Methuen and Co. Ltd. New York: E. P. Dutton and Co. Inc. 